\title{
Fabry Nephropathy in a Young Female Patient Presenting with Only Urinary Mulberry Bodies Treated with Chaperone Therapy
}

\author{
Tsugumi Fukunaga ${ }^{a, b}$ Shingo Nakayama ${ }^{a}$ Takuo Hirose ${ }^{a, c, d}$ \\ Kishin Muramatsu ${ }^{a}$ Akari Endo $^{a}$ Yuka Miyake ${ }^{a, b}$ Go Anan ${ }^{e}$ \\ Ikuko Oba-Yabana ${ }^{a}$ Atsuhiro Kanno ${ }^{f}$ Hannah Nakamura ${ }^{a}$ \\ Junichi Tani $^{a}$ Kimitoshi Nakamurag Kazuhiro Sumitomo ${ }^{f}$ \\ Wako Yumura ${ }^{a}$ h Katsutoshi Furukawa $^{f}$ Takefumi Mori ${ }^{a}$ d
}

\begin{abstract}
aDivision of Nephrology and Endocrinology, Faculty of Medicine, Tohoku Medical and Pharmaceutical University, Sendai, Japan; 'b Department of Nephrology, Japan Self-Defense Forces Sendai Hospital, Sendai, Japan; 'Department of Endocrinology and Applied Medical Science, Tohoku University Graduate School of Medicine, Sendai, Japan; dDivision of Integrative Renal Replacement Therapy, Faculty of Medicine, Tohoku Medical and Pharmaceutical University, Sendai, Japan; 'Department of Urology, Tohoku Medical and Pharmaceutical University, Sendai, Japan; ${ }^{f}$ Division of Community Medicine, Tohoku Medical and Pharmaceutical University, Sendai, Japan; ${ }^{9}$ Department of Pediatrics, Graduate School of Medical Sciences, Faculty of Life Sciences, Kumamoto University, Kumamoto, Japan; hDepartment of Nephrology, Tokyo Metropolitan Geriatric Hospital, Tokyo, Japan
\end{abstract}

Keywords

Fabry disease $\cdot$ Mulberry bodies · Chaperone therapy · Enzyme replacement therapy

\begin{abstract}
Fabry disease (FD) is an X-linked disorder of the sphingolipid metabolism, caused by deficiency or decreased activity of $\alpha$-galactosidase A. We report a rare case of Fabry nephropathy (FN) in a 21-year-old Japanese female patient presenting with only urinary mulberry bodies; she was treated with pharmacological chaperone therapy (PCT) after renal biopsy. The patient underwent a detailed examination because her mother was diagnosed with FD in the Division of Community Medicine of our hospital. She did not have renal dysfunction or proteinuria, and only mulberry bodies were detected in the urine. The activity of $\alpha$-galactosidase A was low, and genetic analysis revealed the R301Q mutation. A percutaneous renal biopsy was performed, and the findings revealed enlargement and vacuolation of glomerular podocytes by
\end{abstract}


Fukunaga et al.: FN Presenting with Only Urinary Mulberry Bodies Treated with Chaperone Therapy

light microscopy, and myelin and zebra bodies were detected in podocytes by electron microscopy. She was diagnosed with FN by renal biopsy and gene analysis. PCT was selected as the treatment to prevent cardiac events and renal dysfunction. The present case suggests that renal biopsy may be necessary even for young women with only mulberry bodies for the diagnosis of FN. It could be useful to evaluate the effect of treatment using the counts of mulberry bodies in the urine. In addition, due to its oral administration, PCT may be suitable for patients who are unable to visit the hospital frequently.

(C) 2021 The Author(s).

Published by S. Karger AG, Basel

\section{Introduction}

Fabry disease (FD) is a poor prognostic disease of the glycosphingolipid metabolism with $\mathrm{X}$-linked disorder caused by deficiency or reduced activity of $\alpha$-galactosidase ( $\alpha$-GAL). FD is a lysosomal storage disorder caused by mutations in the $\alpha$-GAL A-encoding gene on the $\mathrm{X}$ chromosome. FD leads to major disease manifestations, including acroparesthesia, angiokeratoma, hypohidrosis, corneal opacities, and progressive vascular diseases of the kidney and heart [1].

Male patients with FD are classified into 2 categories: the classical and late-onset types, while female patients with FD exhibit heterogeneous disease expression [2, 3]. It is challenging to identify female patients with FD with late-onset type mutations, since there are no overt symptoms during childhood. In addition, FD is reported to progress more slowly in female patients than in male patients [4]. Male patients with FD with complete deficiency of $\alpha$-GAL A activity show severe symptoms, whereas female patients with FD with residual $\alpha$-GAL A activity show symptoms restricted to the heart or kidney, even at a relatively old age [5]. Female patients with FD are at a significant risk of vital organ dysfunction, such as cardiac events and renal dysfunction [6]. It is rare for young female patients with FD with only mulberry bodies to be diagnosed with Fabry nephropathy (FN) via renal biopsy. There is limited information about the cases treated with pharmacological chaperone therapy (PCT) as it is a newly developed treatment for FD [7]. Here, we report a case of FN in a young female patient presenting with only urinary mulberry bodies, who was eventually treated with PCT.

\section{Case Report}

A 21-year-old Japanese woman underwent a detailed examination after her mother was diagnosed with FD. She did not have renal dysfunction or proteinuria, and only mulberry bodies were detected in her urine. FD was observed in the maternal family. Her mother had leg pain and was treated with enzyme replacement therapy (ERT). Her maternal grandfather developed cardiac failure and was undergoing hemodialysis (Fig. 1). Therefore, FD was strongly suspected. Gene analysis showed that the R301Q mutation was found in exon 6 . The serum $\alpha$-GAL A activity of the patient was 8.3 AgalU (normal range $>20.0$ AgaIU). Based on these results, the patient was diagnosed with FD. Physical examination did not reveal acroparesthesia, angiokeratomas, hypohidrosis, or corneal opacities. Since mulberry bodies were continuously detected in the urine, she was introduced to our division and admitted for renal biopsy. On admission, her height was $155.0 \mathrm{~cm}$, and her body weight was $39.9 \mathrm{~kg}$. Her body temperature was $36.5^{\circ} \mathrm{C}$, blood pressure was $103 / 62 \mathrm{~mm} \mathrm{Hg}$, and heart rate was 64 beats/ min with sinus rhythm. Clinical laboratory data are as follows: white blood cell count, $3.6 \times$ 


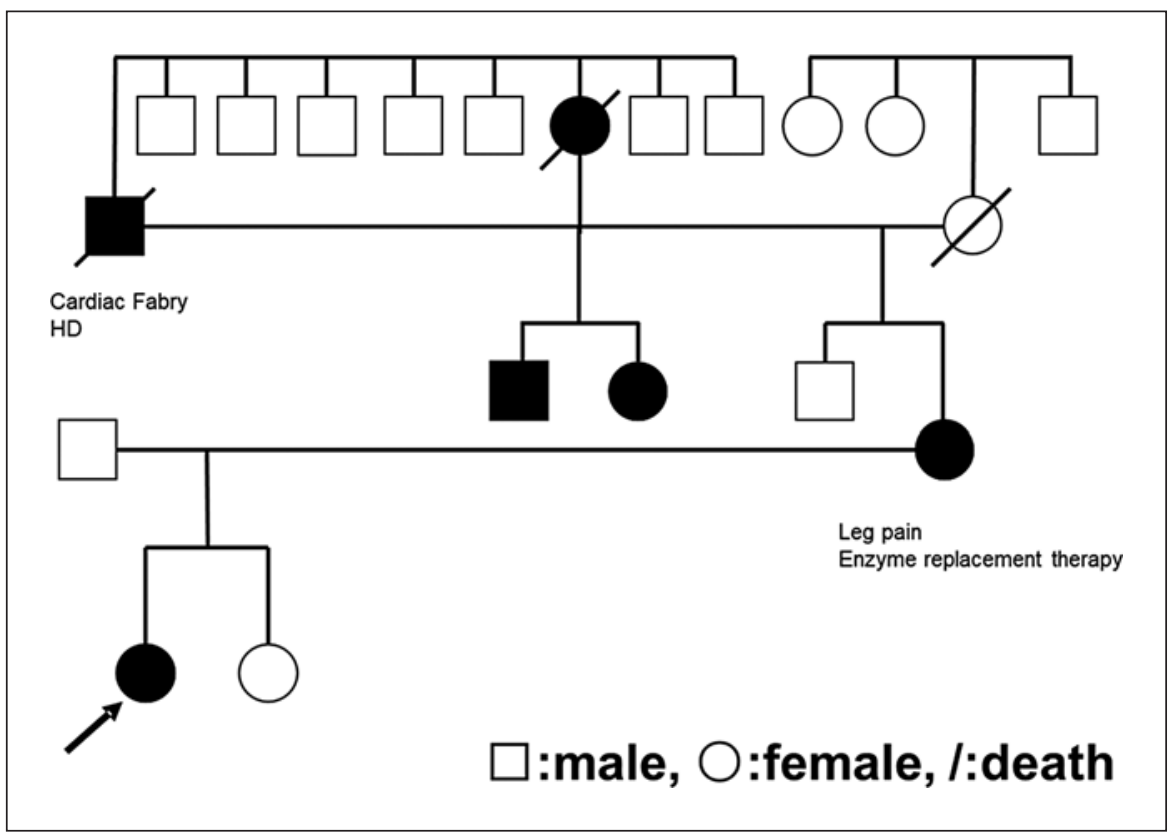

Fig. 1. Family tree. The black arrow indicates the proband. The proband's mother had leg pain and was treated with enzyme replacement therapy. Her grandfather was diagnosed with cardiac Fabry disease and was undergoing HD. HD, hemodialysis.

$10^{3} / \mu \mathrm{L}$ with $43.1 \%$ neutrophils; hemoglobin level, $12.2 \mathrm{~g} / \mathrm{dL}$; platelet count, $18.5 \times 10^{4} / \mu \mathrm{L}$; serum creatinine, $0.54 \mathrm{mg} / \mathrm{dL}$; estimated glomerular filtration rate, $127 \mathrm{~mL} / \mathrm{min} / 1.73 \mathrm{~m}^{2}$; blood urea nitrogen, $7 \mathrm{mg} / \mathrm{dL}$; total protein without M-protein, $6.8 \mathrm{~g} / \mathrm{dL}$; albumin, $3.6 \mathrm{~g} / \mathrm{dL}$; C-reactive protein, $0.01 \mathrm{mg} / \mathrm{dL}$; brain natriuretic peptide, $21.5 \mathrm{pg} / \mathrm{mL}$; immunoglobulin $\mathrm{G}$, $1,040 \mathrm{mg} / \mathrm{dL}$; immunoglobulin A, $100 \mathrm{mg} / \mathrm{dL}$; and immunoglobulin M, $83 \mathrm{mg} / \mathrm{dL}$. Serum complement factors 3 and 4 and complement hemolytic activity were normal. Antinuclear antibodies, rheumatoid factor, and anti-neutrophil cytoplasmic antibodies were negative. Her urinalysis revealed proteinuria $0.02 \mathrm{~g} / \mathrm{g}$ creatinine, $<1 \mathrm{red}$ blood cell/high-power field, $\mathrm{N}$-acetyl- $\beta$-D-glucosaminidase $2.1 \mathrm{U} / \mathrm{L}, \beta 2$-microglobulin $188 \mu \mathrm{g} / \mathrm{L}$, and mulberry bodies (Fig. 2). Echocardiography showed the following findings: left ventricular end-diastolic diameter, $40.7 \mathrm{~mm}$; left ventricular end-systolic diameter, $27.9 \mathrm{~mm}$; ejection fraction, $59.8 \%$; interventricular septal thickness, $6.1 \mathrm{~mm}$; left ventricular posterior wall thickness, $6.0 \mathrm{~mm}$; $\mathrm{E} / \mathrm{A}$ ratio, 2.32; and E/e' 7.4. Left ventricular wall motion abnormalities and valvular disease were not observed. There were no findings suggestive of myocardial or cerebrovascular damage on cardiac and brain magnetic resonance imaging. We performed a percutaneous renal biopsy and obtained the following findings (Fig. 3a-e): the renal biopsy specimens contained 40 glomeruli with no tubulointerstitial changes, but all showed enlarged and vacuolated podocytes under light microscopy. Myelin bodies and zebra bodies were detected in podocytes by electron microscopy. The immunofluorescence staining results were negative. Furthermore, we examined the renal biopsy specimens using low-vacuum scanning electron microscopy (LV-SEM). LV-SEM (Hitachi Tabletop microscope TM4000; Hitachi High-Technologies Corp., Tokyo, Japan) enabled the analysis of the three-dimensional structure of renal biopsy paraffin sections that were stained with Pt-blue [8]. In the new three-dimensional approach, we confirmed the findings of enlarged and vacuolated podocytes (Fig. 3f). Based on the results of renal biopsy, the patient was diagnosed with FN. After diagnosis, she started treatment with PCT to prevent cardiac events and renal dysfunction. 


\section{Case Reports in Nephrology and Dialysis}

\begin{tabular}{l|l}
\hline Case Rep Nephrol Dial 2021;11:355-361 \\
\hline DOI: 10.1159/000520157 & $\begin{array}{l}\text { @ 2021 The Author(s). Published by S. Karger AG, Basel } \\
\text { www.karger.com/cnd }\end{array}$ \\
\hline
\end{tabular}

Fukunaga et al.: FN Presenting with Only Urinary Mulberry Bodies Treated with Chaperone Therapy

Fig. 2. Mulberry bodies in the urine.
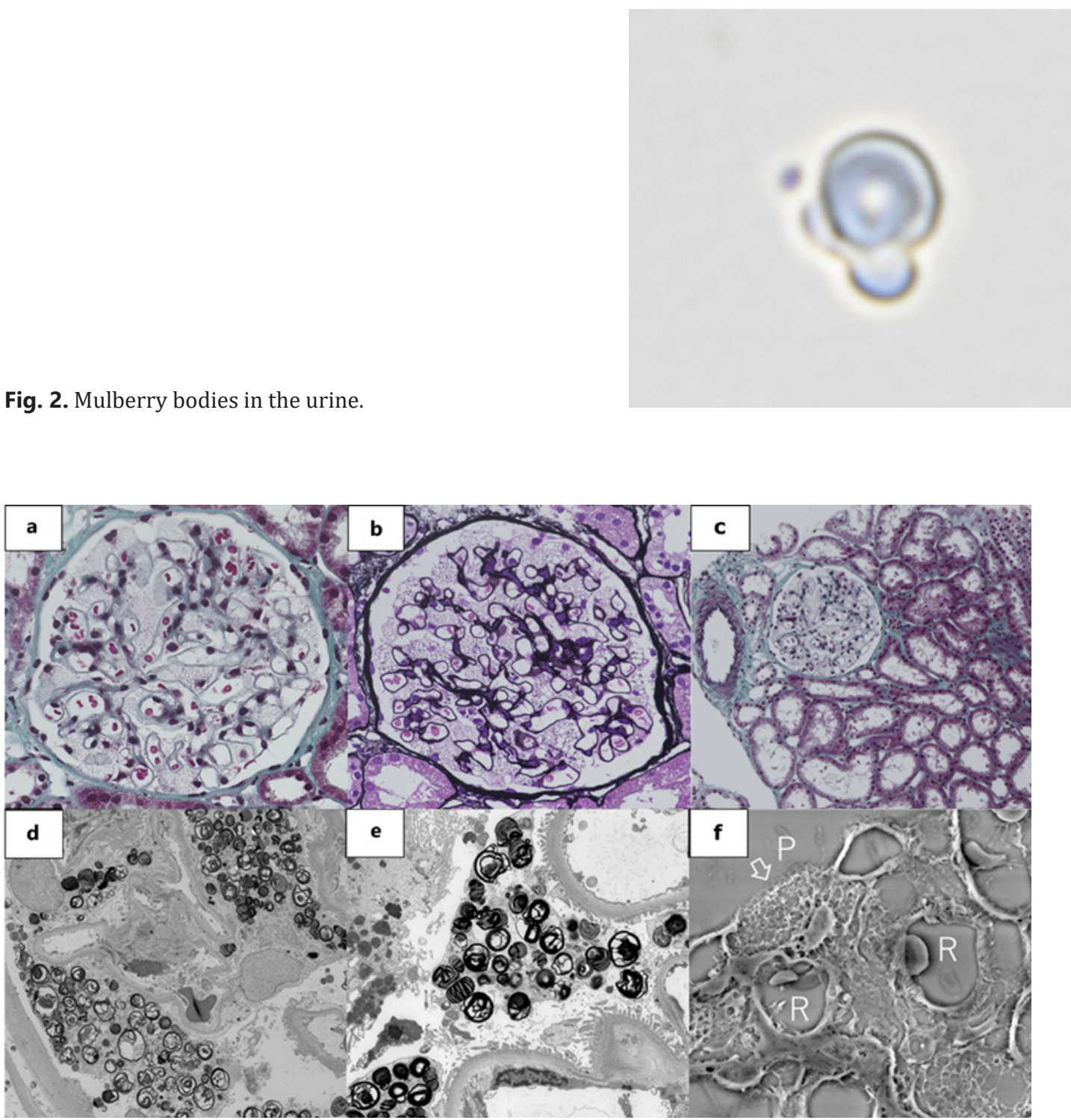

Fig. 3. Representative light microscopy and electron microscopy on the kidney biopsy specimen. a, b Enlarged and vacuolated podocytes observed via light microscopy (a: elastica-Masson stain, original magnification, $\times 400$; b: periodic acid methenamine silver stain, original magnification, $\times 400$ ). $\mathbf{c}$ No tubulointerstitial changes observed via light microscopy (elastica-Masson stain, original magnification, $\times 100$ ). d, e Electron microscopy showing osmiophilic lamellar inclusions, with a concentric pattern called myelin bodies, or with elongated stripes called zebra bodies mainly in the podocytes (d: original magnification, $\times 2500$; e: original magnification, $\times 5000)$. $f$ Low-vacuum scanning electron microscopy showing enlarged and vacuolated podocytes three dimensionally. Podocytes (P, white arrow) and red blood cells (R) are shown (f: Pt-blue stain, original magnification, $\times 2500)$.

\section{Discussion}

We encountered a case of FN in a young female patient presenting with only urinary mulberry bodies, who was eventually treated with PCT. A previous study demonstrated that the condition of male patients with FD progressed more rapidly than that of female patients with FD [4]. However, female patients with FD have a high risk of cardiac events and renal 
dysfunction [6]. Therefore, female patients with FD need to be treated earlier to prevent cardiac events and renal dysfunction.

More than 700 FD-causing mutations have been identified in genes encoding human $\alpha$-GAL A. The range of phenotypes for $\alpha$-GAL A mutations can vary widely, depending on the individual genotype in patients with FD [9]. The most commonly identified pathogenic mutations in Japanese FD are M296I, R227X, R112H, R112C, and R301Q [2]. In the present case, the R301Q mutation was found in exon 6. This is one of the mutations of variant phenotype FD and is found in patients with FD of both the classic and later-onset types [2]. Yamamoto et al. [3] reported the clinical diversity in patients with FD with the R301Q mutation, and their clinical manifestations were markedly divergent despite the same genetic abnormality. According to the reported cases by Yamamoto et al. [3], FN was diagnosed by renal biopsy in 3 ( 1 male and 2 female patients) out of 10 cases. Two female patients without renal dysfunction were diagnosed with FN by renal biopsy; however, they were over 40 years old [3]. The present case was of a female FN patient diagnosed at a young age presenting with only mulberry bodies in her urine.

Mulberry bodies, which are caused by the accumulation of globotriaosylceramide on the epithelial cells of the distal convoluted tubules, are specific to FD [10]. The diagnostic accuracy may vary depending on the ability and experience of the medical technician, and, to the best of our knowledge, no study has described the relationship between the quantities of mulberry bodies and the progression of renal dysfunction in patients with FD or their utility as biomarkers for treatment. However, mulberry bodies are inexpensive and noninvasive biomarkers, and the counts of mulberry bodies may be useful for therapeutic evaluation [11]. Moreover, several case reports have indicated the usefulness of mulberry bodies in the early diagnosis of FN $[10,12]$.

The treatment options for FD include ERT and PCT. ERT was developed in 2001 as a treatment for FD. ERT is based on a chronic, 2-weekly infusion of agalsidase $\alpha$ or $\beta$ [13]. In a previous study, ERT slowed the progression of renal dysfunction, cardiomyopathy, and delayed mortality [14]. However, ERT needs to be applied intravenously, and some patients develop infusion-associated reactions and antibody formation to ERT. Oral migalastat, a pharmacological chaperone, was approved in Germany in 2016. Migalastat is administered orally every other day. Migalastat binds reversibly to the $\alpha$-GAL active site, stabilizes specific mutant forms of the enzyme, and promotes trafficking to lysosomes, where $\alpha$-GAL catabolizes accumulated disease substrates in patients with FD [15]. Migalastat is expected to be an alternative treatment for ERT. However, this therapy is limited to a specific number of mutations. It is estimated that only $35 \%-50 \%$ of patients with FD have migalastat-amenable mutations [7]. Our patient had mutant forms of $\alpha$-GAL that were responsive to migalastat. PCT significantly decreased the left ventricular mass index, which may contribute to a decrease in cardiac events. Left ventricular hypertrophy is associated with cardiac events in patients with FD. Left ventricular mass has been shown to be an important parameter for assessing the cardiac effects of the treatment in patients with FD [15]. Male patients with FD receiving PCT had an increase in leukocyte $\alpha$-Gal A activity, while this was not confirmed in female patients with FD [16]. Therefore, it could be meaningful to measure the change in enzyme activity in WBC for monitoring the therapeutic effect of PCT in patients with FD. In a randomized, active-controlled study, PCT and ERT also had similar effects on renal function [7]. In addition, PCT can avoid infusion-associated reactions and antibody formation [15]. PCT has better potential to improve the quality of life compared to ERT due to its oral administration. Since our case was of a young patient who had difficulty in frequent hospital visits, the treatment had a positive effect on her quality of life.

In summary, although the patient in the present case did not have renal dysfunction and proteinuria, mulberry bodies were detected in her urine. Based on the results of her renal biopsy, the patient was diagnosed with FN at a young age. Young female patients presenting with only mulberry bodies and diagnosed with FN by renal biopsy are rare. The present case

\section{Karger'}


Case Reports

in Nephrology

and Dialysis

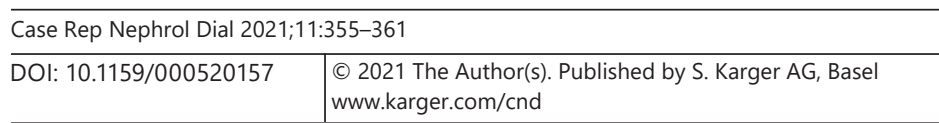

Fukunaga et al.: FN Presenting with Only Urinary Mulberry Bodies Treated with Chaperone Therapy

suggests that renal biopsy can be necessary even for young women presenting with only mulberry bodies to prevent cardiac events and renal dysfunction by early detection of FN. Since the R301Q mutation was found in exon 6, ERT was considered to be a preferable choice for FN according to previous reports $[13,14]$. However, we selected PCT as her treatment as she had difficulty visiting a hospital frequently, and PCT and ERT have similar treatment effects on renal function [7]. It could be useful to assess the effect of treatment using the counts of mulberry bodies in FN patients. Furthermore, due to its oral administration, PCT could be suitable for patients who are unable to visit the hospital regularly.

\section{Statement of Ethics}

This work was approved by the Ethics Committee of the Tohoku Medical and Pharmaceutical University (2021-4-016). The research was conducted ethically in accordance with the World Medical Association Declaration of Helsinki. Written informed consent was obtained from the patient to publish this case report and any accompanying images.

\section{Conflict of Interest Statement}

The Division of Integrative Renal Replacement Therapy is financially supported by Chugai Pharmaceutical, Terumo, and JMS (T.H. and T.M.). The other authors declare that no conflicts of interest exist.

\section{Funding Sources}

This work was supported in part by Grants-in-Aid for Scientific Research (19H03677, 19K21596, and 20K08612) from the Ministry of Education, Culture, Sports, Science, and Technology of Japan (MEXT) and the Takeda Research Foundation.

\section{Author Contributions}

T.F. contributed to acquisition of data and writing of the manuscript. S.N. and W.Y. provided supervision and mentorship. T.H., G.A., K.N., and T.M. revised the article critically for important intellectual content. All authors read and approved the final manuscript.

\section{Data Availability Statement}

The data used to support the findings of this work are available from the corresponding author on request (contact S.N., nakayama-s@tohoku-mpu.ac.jp).

\section{References}

1 Ries M, Ramaswami U, Parini R, Lindblad B, Whybra C, Willers I, et al. The early clinical phenotype of Fabry disease: a study on 35 European children and adolescents. Eur J Pediatr. 2003;162:767-72.

2 Sakuraba H, Tsukimura T, Togawa T, Tanaka T, Ohtsuka T, Sato A, et al. Fabry disease in a Japanese populationmolecular and biochemical characteristics. Mol Genet Metab Rep. 2018;17:73-9. 
3 Yamamoto S, Nagasawa T, Sugimura K, Kanno A, Tatebe S, Aoki T, et al. Clinical diversity in patients with Anderson-fabry disease with the R301Q mutation. Intern Med. 2019;58:603-7.

4 Schiffmann R, Warnock DG, Banikazemi M, Bultas J, Linthorst GE, Packman S, et al. Fabry disease: progression of nephropathy, and prevalence of cardiac and cerebrovascular events before enzyme replacement therapy. Nephrol Dial Transplant. 2009;24:2102-11.

5 Deegan PB, Baehner AF, Barba Romero MA, Hughes DA, Kampmann C, Beck M. Natural history of Fabry disease in females in the Fabry outcome survey. J Med Genet. 2006;43:347-52.

6 Wilcox WR, Oliveira JP, Hopkin RJ, Ortiz A, Banikazemi M, Feldt-Rasmussen U, et al. Females with Fabry disease frequently have major organ involvement: lessons from the Fabry Registry. Mol Genet Metab. 2008;93:11228.

7 Hughes DA, Nicholls K, Shankar SP, Sunder-Plassmann G, Koeller D, Nedd K, et al. Oral pharmacological chaperone migalastat compared with enzyme replacement therapy in Fabry disease: 18-month results from the randomised phase III ATTRACT study. J Med Genet. 2017;54:288-96.

8 Inaga S, Kato M, Hirashima S, Munemura C, Okada S, Kameie T, et al. Rapid three-dimensional analysis of renal biopsy sections by low vacuum scanning electron microscopy. Arch Histol Cytol. 2010;73:113-25.

9 Čerkauskaitė A, Čerkauskienė R, Miglinas M, Laurinavičius A, Ding C, Rolfs A, et al. Genotype ${ }^{-}$Phenotype Correlation in a New Fabry-Disease-Causing Mutation. Medicina. 2019;55:122.

10 Shimohata H, Maruyama H, Miyamoto Y, Takayasu M, Hirayama K, Kobayashi M. Urinary mulberry cells and mulberry bodies are useful tool to detect late-onset Fabry disease. CEN Case Rep. 2017;6:148-51.

11 Aoyama Y, Ushio Y, Yokoyama T, Taneda S, Makabe S, Nishida M, et al. Urinary mulberry cells as a biomarker of the efficacy of enzyme replacement therapy for Fabry disease. Intern Med. 2020;59:971-6.

12 Nakamichi T, Miyazaki M, Nakayama K, Sato M, Akiu N, Sato T, et al. Fabry's disease discovered with chance urinary mulberry cells: a case report. CEN Case Rep. 2013;2:49-52.

13 Tsuboi K, Yamamoto H. Efficacy and safety of enzyme-replacement-therapy with agalsidase alfa in 36 treatment-naïve Fabry disease patients. BMC Pharmacol Toxicol. 2017;18:43.

14 Beck M, Hughes D, Kampmann C, Larroque S, Mehta A, Pintos-Morell G, et al. Long-term effectiveness of agalsidase alfa enzyme replacement in Fabry disease: a Fabry Outcome Survey analysis. Mol Genet Metab Rep. 2015;3:21-7.

15 Germain DP, Hughes DA, Nicholls K, Bichet DG, Giugliani R, Wilcox WR, et al. Treatment of Fabry's disease with the pharmacologic Chaperone Migalastat. N Engl J Med. 2016;375:545-55.

16 Narita I, Ohashi T, Sakai N, Hamazaki T, Skuban N, Castelli JP, et al. Efficacy and safety of migalastat in a Japanese population: a subgroup analysis of the ATTRACT study. Clin Exp Nephrol. 2020;24:157-66. 